\title{
Febuxostat ameliorates methotrexate-induced lung damage
}

\author{
S.M. Zaki ${ }^{1}{ }^{2}$, G.H.A. Hussein ${ }^{3}$, H.M.A. Khalil' ${ }^{4}$, W.A. Abd Algaleel ${ }^{1}$ \\ ${ }^{1}$ Department of Anatomy and Embryology, Faculty of Medicine, Cairo University, Cairo, Egypt \\ ${ }^{2}$ Fakeeh College for Medical Sciences, Jeddah, Saudi Arabia \\ ${ }^{3}$ Department of Anatomy and Embryology, Faculty of Medicine, Beni Suef University, Beni Suef, Egypt \\ ${ }^{4}$ Department of Veterinary Hygiene and Management, Faculty of Veterinary Medicine, Cairo University, Cairo, Egypt
}

[Received: 17 June 2020; Accepted: 1 July 2020]

Background: The intention of the present study was to assess the structural affection of the lung following methotrexate (MTX) overdose. The proposed underlying mechanisms involved in lung affection were studied. The possible modulation role of febuxostat over such affection was studied.

Materials and methods: Twenty-four rats were divided into three groups: control, MTX-treated, febuxostat-treated. The study was continued for 2 weeks. Lung was processed for histological and immunohistochemical (inducible nitric oxide synthase [iNOS] and cyclooxygenase [COX]-2) studies. Inflammatory markers (tumour necrosis factor alpha [TNF- $\alpha]$, interleukin 1 [IL-1]), Western blot evaluation

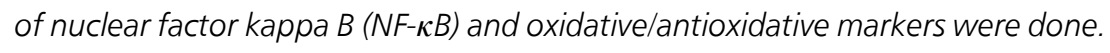
Results: Methotrexate-treated group exhibited inflammatory cellular infiltrations, thickened interalveolar septa, dilated congested blood vessels, extravasated blood, and apoptosis. The collagen fibres content increased 3-fold. MTX induced lung affection through oxidative stress (increase MDA/decrease GSH, SOD) and apoptosis. It induced sterile inflammation through an increase of NF- $\kappa B$ (2-fold), IL-1 (3-fold) and TNF- $\alpha$ (3-fold), COX-2 cells (2.5-fold) and iNOS (6-fold). With the use of febuxostat, the normal lung architecture was observed with a bit thickened interalveolar septum and extravasated blood. The collagen fibres content was minimal. Decrement of oxidative stress and sterile inflammation (COX-2 cells and iNOS were comparable to the control group. NF- $\kappa B$, IL-1 and TNF- $\alpha$ became higher by $34 \%, 64 \%$ and $100 \%)$.

Conclusions: The overdose of MTX displays inflammatory lung affection with residual fibrosis. It induces lung affection through oxidative stress, apoptosis and sterile inflammation. With the use of febuxostat, the normal lung architecture was preserved with a little structural affection or fibrotic residue. Febuxostat exerts its lung protection through its anti-inflammatory and antioxidant features. (Folia Morphol 2021; 80, 2: 392-402)

Key words: febuxostat, methotrexate, lung

\section{INTRODUCTION}

Methotrexate (MTX) has displayed effectiveness in treating several diseases, including rheumatoid arthritis, psoriatic arthritis, systemic lupus erythematosus, inflammatory bowel disease, psoriasis, and small-vessel vasculitis [11, 32]. It is commonly used 
as a treatment for some types of cancer, including breast, ovaries, brain, and leukaemia [26]. It is used as an alternative to surgical management of ectopic pregnancy in selected patients with small, unruptured tubal pregnancies [34]. In addition, it is recommended for induction and maintenance of remission in Crohn's disease [13].

The therapeutic application of MTX is usually limited by its severe toxicity [38]. It is involved as a causative agent in lung toxicity $[9,33]$. The prevalence of MTX-related lung disease and MTX-related interstitial lung disease (in rheumatoid arthritis) are $7.6 \%$ and $11.6 \%$, respectively $[4,9]$.

Several mechanisms have been studied to recognise the mechanism of MTX-induced lung damage. One of these mechanisms is oxidative stress [2]. MTX interferes with the antioxidant defence enzymes, depletes glutathione content, and causes lipid peroxidation [2]. Diminution of antioxidant defences enhances the production of reactive oxygen species (ROS) that result in parenchymal lung injury and interstitial, alveolar fibrosis [24]. Other possible mechanism of MTX-induced lung damage is inflammatory reaction of MTX. MTX increases the levels of interleukin 1 beta (IL-1 $\beta$ ), and tumour necrosis factor alpha (TNF- $\alpha$ ), which are indicators of inflammatory response [1]. Adding, overdose of MTX can lead to proinflammatory cytokine release [19].

Febuxostat is a selective xanthine oxidase inhibitor that is used to reduce urate levels in diseases that involve hyperuricemia such as gout and tumour lysis syndrome [28, 31]. The focus on xanthine oxidase inhibitors has increased due to their anti-inflammatory, antioxidant and immune-modulatory features which might be beneficial in the treatment of different auto-inflammatory diseases [27]. Febuxostat showed anti-inflammatory effects in different experimental models [15]. It has an antioxidative stress effect and suppressed ROS production in the rat model of renal ischemia-reperfusion injury [36].

The intention of the present study was to assess the structural affection of the lung following MTX overdose. The proposed underlying mechanisms involved in lung affection were studied. The possible modulation role of febuxostat over such affection was studied.

\section{MATERIALS AND METHODS}

\section{Animals}

Twenty-four Sprague-Dawley adult albino rats were used. The sample size was based according to resource equation method $[10,16]$. The rats were housed in a temperature of $22 \pm 2^{\circ} \mathrm{C}$, relative humidity of $55 \pm 5 \%$, and $12 / 12 \mathrm{~h}$ light/dark cycle. The study was conducted in Experimental Animal Centre, Cairo University. The experiment was carried out in accordance to ARRIVE guidelines (Animal Research: Reporting of In-Vivo Experiments) with the approval of the local IACUC Research Ethics Committee and according to ethical standards of National Institutes of Health guide for care and use of Laboratory Animals ( $8^{\text {th }}$ edition, revised in 2011).

\section{Experimental design}

The rats were divided into three groups. Each group consisted of 8 rats:

- Control group: $100 \mathrm{mg} / \mathrm{kg}$ physiological saline/ /intraperitoneally;

- MTX-treated group;

- Febuxostat-treated group (concomitant MTX + febuxostat).

\section{Test materials}

Methotrexate was obtained from Pfizer Pharmaceutical Company and Chemical industries (Egypt) and given as a single dose of $20 \mathrm{mg} / \mathrm{kg}$, intraperitoneally [2]. The used dose of MTX was a high dose to evaluate the role of ROS formation and apoptosis [21].

Febuxostat was obtained from Hikma Pharmaceutical Co. (Egypt). It was dissolved in $0.9 \%$ saline and given at a dose of $15 \mathrm{mg} / \mathrm{kg}$, orally: 2 drops of Tween 80 [15] for 14 successive days.

\section{General toxicological profile}

The general toxicological data were recorded including food and water consumption, health status, body weight and behavioural measures.

\section{Behavioural measures (anxiety-like behaviour)}

Two days before the end of the experiment, the rats were subjected to behavioural observation to measure anxiety-like behaviour using two behavioural tests. The rats were accustomed to the presence of the experimenter before the behavioural observation. The tests were carried out between 9 a.m. and 12 p.m. for 2 days.

Light/dark box. This test based on the innate fear of the rat to bright spaces. It is composed of a box divided into equal compartment, bright and dark compartments separated by a partition with a door. The rat was placed at the bright compartment and 
allowed to explore for $5 \mathrm{~min}$. Frequency of entry into dark and light compartments and the time spent in these compartments were measured [7].

Elevated plus maze [37]. This test is based on the rat's conflict between exploring novel places, and avoiding heights and dangerous places. It is composed of wooden two open and enclosed arms connected by central platform. The apparatus elevated $60 \mathrm{~cm}$ above the ground. The rats were placed in the open arm and allowed to explore for $5 \mathrm{~min}$. Frequency of entry to the open and closed arms as well as the time spent in each arm were measured.

\section{Tissue sampling}

The lung was dissected and fixed immediately in $10 \%$ formalin saline. To randomise selection, the entire lung was cut starting at the superior border; every $10^{\text {th }}$ section ( $5 \mu \mathrm{m}$ thick) was put aside for staining.

\section{Preparation of tissue extracts}

A portion of lungs was homogenised in 10 volumes (1:10; w/v) of ice-cold $10 \mathrm{mM}$ phosphate buffered saline (PBS, pH 7.4) in an Ultra Turrax tissue homogenizer for $30 \mathrm{~s}$. Homogenates were centrifuged at $10,000 \mathrm{rpm}$ for $10 \mathrm{~min}$ at $4^{\circ} \mathrm{C}$. The supernatant was pipetted into clean centrifuge tubes and stored in aliquots $\left(-80^{\circ} \mathrm{C}\right)$ until analysis [12].

\section{Light microscopic study}

\section{Haematoxylin and eosin stain}

The technique of haematoxylin and eosin stain was done according to Suvarna et al. [35].

\section{Masson's trichrome stain [35]}

The paraffin sections were dewaxed, rehydrated then stained in acid fuchsin solution for $5 \mathrm{~min}$, rinsed in distilled water, placed in phosphomolybdic acid solution for $3 \mathrm{~min}$, washed in distilled water, stained with methyl blue solution for 2-5 $\mathrm{min}$, rinsed in distilled water, and treated in acetic acid for $2 \mathrm{~min}$. Finally, the sections were dehydrated in absolute alcohol, cleared in xylol, and mounted in Canada balsam. The nuclei appeared dark red, the cytoplasm appeared pale red and, the collagen fibres appeared blue.

\section{Immunohistochemistry [30]}

Immunohistochemical staining using the streptavidin-biotin-peroxidase technique for inducible nitric oxide synthase (iNOS), and cyclooxygenase (COX)-2 (markers of inflammation). Briefly, 10 sections/group were deparaffinised, rehydrated, and rinsed in tap water, treated with $3 \%$ hydrogen peroxide for $10 \mathrm{~min}$ then, immersed in antigen retrieval solution. Non-specific protein binding was blocked by incubating the sections in $10 \%$ normal goat serum in phosphate buffer solution (PBS). Then, the sections were incubated in a humid chamber at $4^{\circ} \mathrm{C}$ with primary anti-iNOS antibody (rabbit polyclonal antibody, 1:100 dilution, ab15323, Abcam, Cambridge, Massachusetts, USA), anti-nuclear factor kappa B (NF-кB) antibody (rabbit polyclonal anti-rat antibody against $\mathrm{P} 65$ subunit of NF-kB; 1:20 dilution, ab86299, Abcam, Cambridge, Massachusetts, USA) and anti-COX-2 (rabbit polyclonal antibody, 1:100 dilution, ab15191, Abcam, Cambridge, Massachusetts, USA) overnight. After washing in PBS, the corresponding biotinylated secondary antibody was added to lung sections for $1 \mathrm{~h}$ at room temperature. Streptavidin peroxidase was added for 10 min and then washed in PBS. Finally, the sections were counterstained by Mayer's haematoxylin. For negative control sections, the primary antibodies were excluded. All the slides were assessed in triplicates to confirm the accuracy of the obtained results.

\section{Oxidative/antioxidative markers}

Lung lipid peroxidation. Malondialdehyde (MDA), a marker for lipid peroxidation, was measured by monitoring thiobarbituric reactive substances formation. Briefly, $500 \mathrm{~mL}$ of lung homogenate was added to $200 \mathrm{~mL}$ of PBS (10 Mm, pH 7.4) and $500 \mu \mathrm{L}$ of heat trichloroacetic acid-butylated hydroxytoluene ( $20 \%$ TCA, 1\% BHT) solution. The resultant was mixed and centrifuged at $3000 \mathrm{rpm}$ for $10 \mathrm{~min}$ at $4^{\circ} \mathrm{C}$. To $800 \mathrm{~mL}$ of supernatant, $160 \mu \mathrm{L}$ of $0.6 \mathrm{M} \mathrm{HCL}$ and $640 \mu \mathrm{L}$ of $1.73 \%$ thiobarbituric acid were added. This suspension was mixed and heated in a boiling water bath for $15 \mathrm{~min}$. After cooling, the thiobarbituric reactive substances were measured in the supernatant at $530 \mathrm{~nm}$ against a blank containing all reagents except the tissue homogenate. The concentration of MDA was calculated and expressed in Nano-moles per milligram of protein.

Lung SOD activity. Superoxide dismutase activity (SOD) was determined at room temperature (RT) according to the modified Misra and Fridovich's method [23]. $5 \mu \mathrm{L}$ of $10 \%$ lung homogenate was added to $1965 \mu \mathrm{L}$ of sodium carbonate buffer and to $10 \mu \mathrm{L}$ of bovine catalase. $20 \mu \mathrm{L}$ of $30 \mathrm{mM}$ epinephrine (dissolved in $0.05 \%$ acetic acid) was added to the mixture. Superoxide dismutase activity was measured at 
$480 \mathrm{~nm}$ for $5 \mathrm{~min}$ on a spectrophotometer. The activity was expressed as the amount of the enzyme that inhibits the oxidation of epinephrine by $50 \%$, which is equal to $1 \mathrm{U} / \mathrm{mg}$ of protein.

Lung glutathione (GSH). Glutathione was measured spectrophotometrically in the bronchoalveolar lavage fluid (BALF) and lung tissue [29]. GSH was measured by adding the standard or sample to $100 \mu \mathrm{L}$ of a 1:1 mixture of $3 \mathrm{U} / \mathrm{mL}$ glutathione reductase

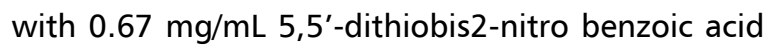
(DTNB). The reaction was initiated by the addition of $20 \mu \mathrm{L}$ of $0.67 \mathrm{mg} / \mathrm{mL}$ nicotinamide adenine dinucleotide phosphate (NADPH) and the increase in absorbance at $412 \mathrm{~nm}$ was monitored by using a commercial kit (Biodiagnostic, Cairo, Egypt). Values measured in BALF were normalised to urea, values in lung tissue were normalised to protein content. For in vitro samples both the media and lysate were normalised to the lysate protein. The limit of detection for GSH was $0.2 \mu \mathrm{M}$. The concentration of the lung GSH was calculated using the standard curve and expressed per mg of protein. The level of epithelial lining fluid GSH (ELF GSH) was expressed in $\mu \mathrm{M}$.

\section{Western blot assay}

Nuclear factor kappa B was estimated by the enzyme-linked immunosorbent assays (ELISA), according to the manufacturer's guide (R\&D System Inc.). Lung tissue was standardised $(1: 10)$ in saline and stockpiled at $-80^{\circ} \mathrm{C}$. Models were preserved with cell lysis buffer, adjusted with PMSF and PIC former to the measures. Samples were extra watered down in assay buffer. After pipetting $100 \mu \mathrm{L}$ assay buffer, $100 \mu \mathrm{L}$ of sample/standard were supplemented to the pits.

The plate was incubated at RT for $1 \mathrm{~h}$. The wells were splashed 5 times with rinse buffer. After $1 \mathrm{~h}$ incubation, the plate was splashed 5 times with shower buffer and $100 \mu \mathrm{L}$ of conjugate were supplemented to all wells. The plate was vacuum-packed and incubated for 30 min at RT. Well matters were let down and rinsed 5 times in buffer. $100 \mu \mathrm{L}$ of substrate was auxiliary to all wells and colour endorsed to progress. $30 \mathrm{~min}$ later, halt solution was substituted. The plate was delivered on an ELISA Plate Reader (OD $450 \mathrm{~nm}$ ). Rectilinear average curves were created in assay buffer to estimate the values of NF-KB.

\section{Image analysis and morphometric measurements}

The area per cent of collagen fibres and immune expression of iNOS, and COX-2 were done using Leica
LAS V3.8 image analyser computer system (Switzerland). The measurements were obtained by an independent blinded observer. The data was obtained in ten non-overlapping microscopic fields taken randomly from each slide and were examined within the standard measuring frame.

\section{Biochemical assay}

The serum level of the inflammatory markers TNF- $\alpha$, and IL- 1 were assayed by the commercially ELISA kits supplied by Biopsies, China according to manufacturer instructions.

\section{Protein assay}

The protein concentration in the lung homogenate was measured by Bradford's method using bovine serum albumin as standard [8].

\section{Statistical analysis}

Statistical analysis was performed using statistical package for the social sciences (SPSS) version 21.0 (IBM Corporation, Somers, NY, USA) statistical software. Data were expressed as means \pm standard deviation (SD). Statistical evaluation was done using one-way analysis of variance (ANOVA) followed by Bonferroni pairwise comparisons. Significance was considered when the $p$-value was less than 0.05 .

The percentage of increase or decrease (difference) of all study parameters were calculated with the following formula: Percentage of difference $=$ $=($ Mean difference value between two groups $) /($ Value of the compared group) $\times 100$.

\section{RESULTS}

\section{The general toxicological data}

No mortalities were noticed in the studied groups. The food and water intake and health status were relatively excellent.

At the beginning of study, the body weight was $150 \pm 3.6 \mathrm{~g}$. By the end of study, the body weight of MTX-treated group decreased by $22 \%$ compared to the control group. With use of febuxostat, the body weight became $12 \%$ less than the control group (Table 1 ).

\section{Behavioural measures (anxiety-like behaviour)}

Light/dark box. There was non-significant difference among the different groups concerning time spent in the dark or light compartments. A significant increase $(130 \%)$ in the number of entries of the MXT-treated rats into the dark compartment as compared to the control 
Table 1. Body weight in the different groups at the end of study

\begin{tabular}{lcc}
\hline Group & Body weight [g] & Versus group \\
\hline Control & $185 \pm 5$ & \\
MTX-treated & $145 \pm 5$ & $\begin{array}{c}\text { Control* } \\
\text { Febuxostat-treated* }\end{array}$ \\
Febuxostat-treated & $162.6 \pm 2.5$ & Control $^{*}$ \\
& & MTX-treated* \\
\hline
\end{tabular}

*P-value significant. Data are shown as mean \pm standard deviation. The body weight at the beginning of the study was $150 \pm 3.6 \mathrm{~g}$. MTX - methotrexate

rats. Non-significant entry difference was detected in febuxostat-treated group into dark and light compartments as compared to the control group (Fig. 1).

Elevated plus maze. As comparted to the other groups, MXT-treated rats entered the open arm of the elevated plus maze less frequent and spent less time. They entered more frequent the closed arm of the elevated plus maze and spent more time (Fig. 2A, B).
Febuxostat-treated rats entered more frequent and spent more time in the open arm (Fig. 2C, D).

\section{Structure of lung as revealed by haematoxylin and eosin staining}

The control group presented normal lung morphology. MTX-treated rats exhibited inflammatory cellular infiltrations, thickened interalveolar septa (with oedema), dilated congested blood vessels, extravasated blood, and apoptotic pneumocytes. With the use of febuxostat, normal lung architecture was observed with a bit thickened interalveolar septum and extravasated blood (Figs. 3, 4).

\section{The content of collagen fibres}

The collagen fibres were minimum in the control group. The fibres content increased three-fold in MTX-treated group when compared to the control group. Much improvement was noticed in febux-
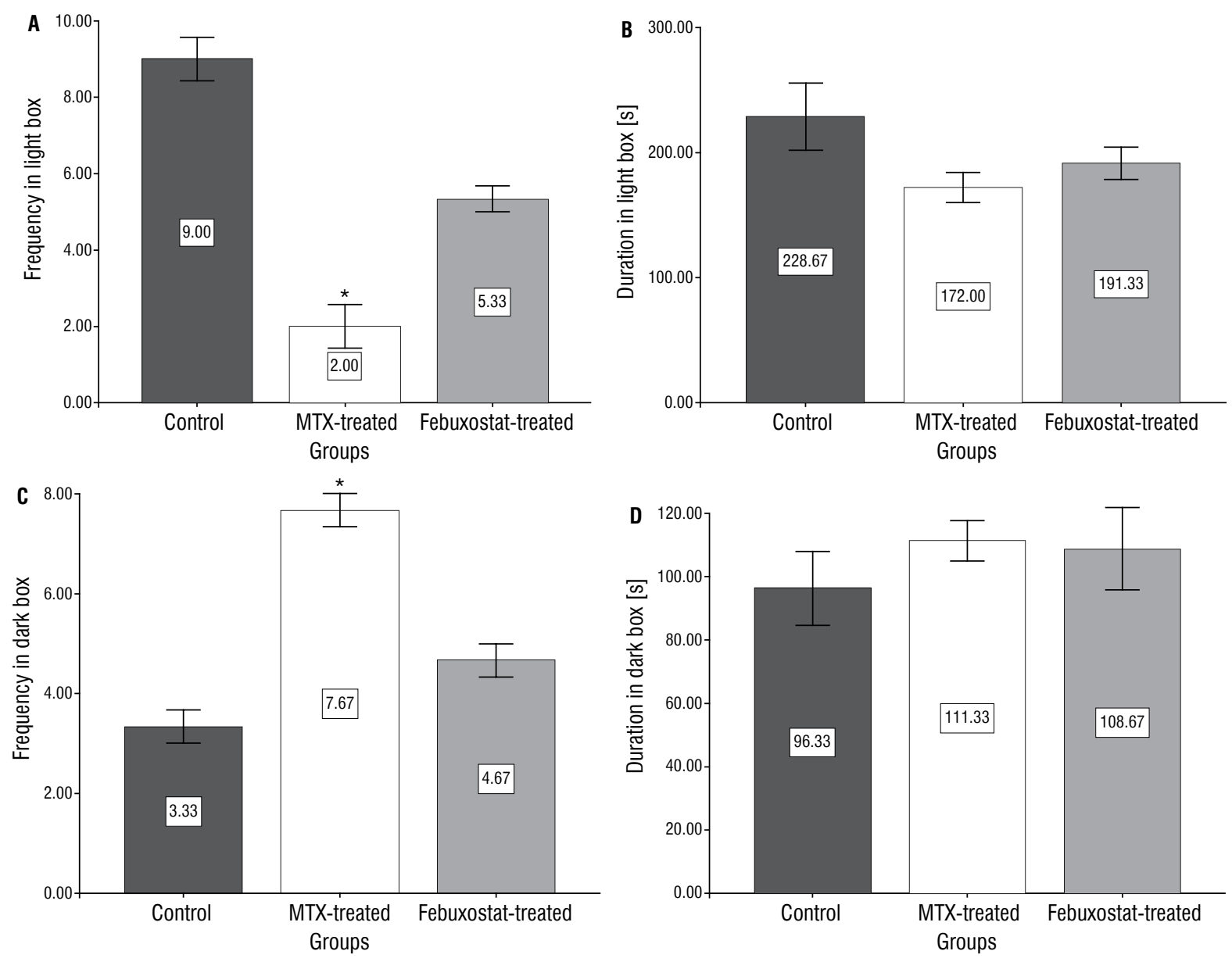

Figure 1. Anxiety-like behaviour in the light/dark box test; A. Frequency of entry into the light compartment; B. Time spent in the light compartment; C. Frequency of entry into the dark compartment; D. Time spent in the dark compartment; ${ }^{*}$ Significant from control group; $p=0.006$. 

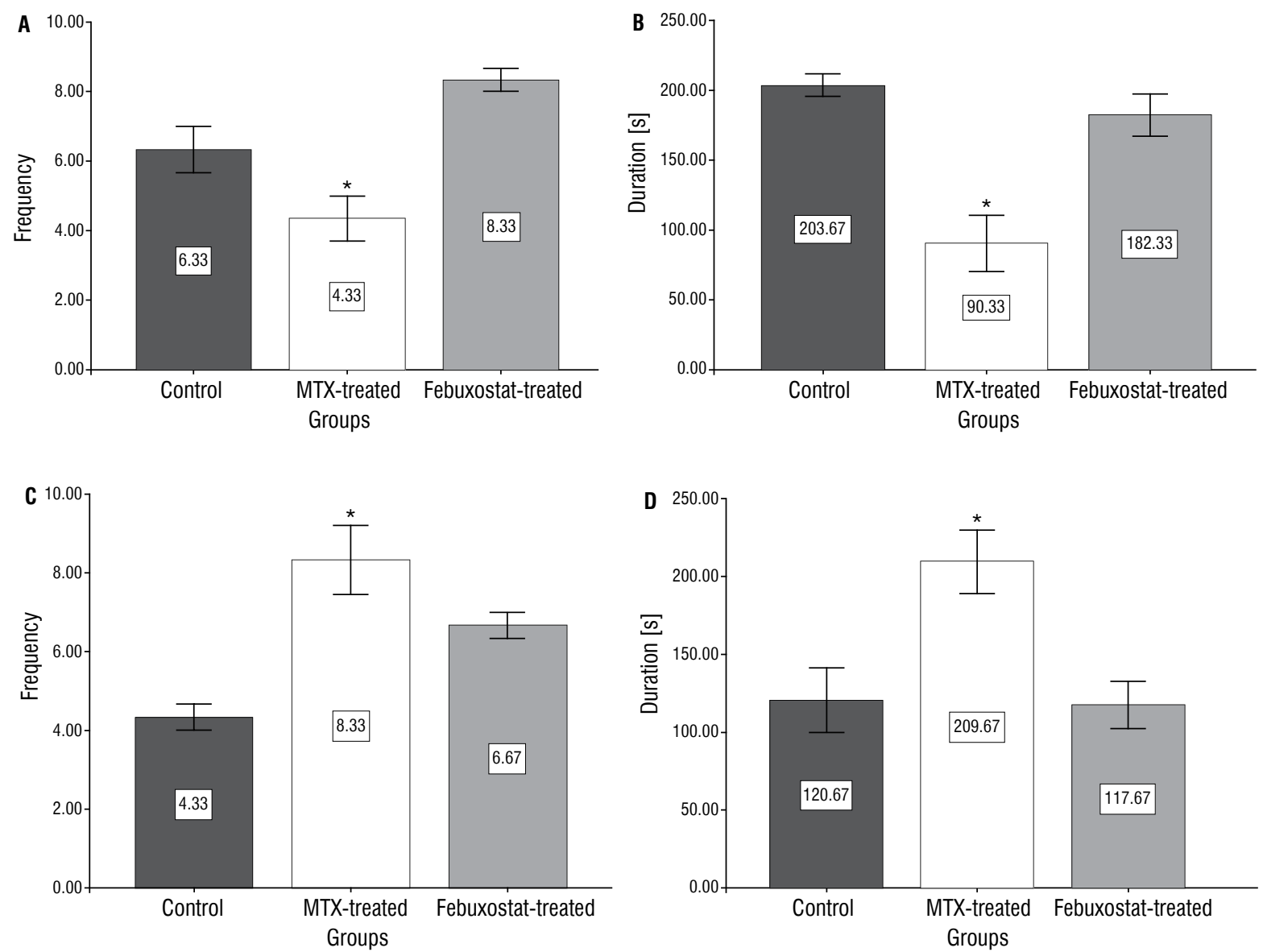

Figure 2. Anxiety-like behaviour in the elevated plus maze teat; A. Open arm entry; B. Open arm duration; C. Close arm entry; D. Close arm duration; *Significant from control group; $p=0.000$.

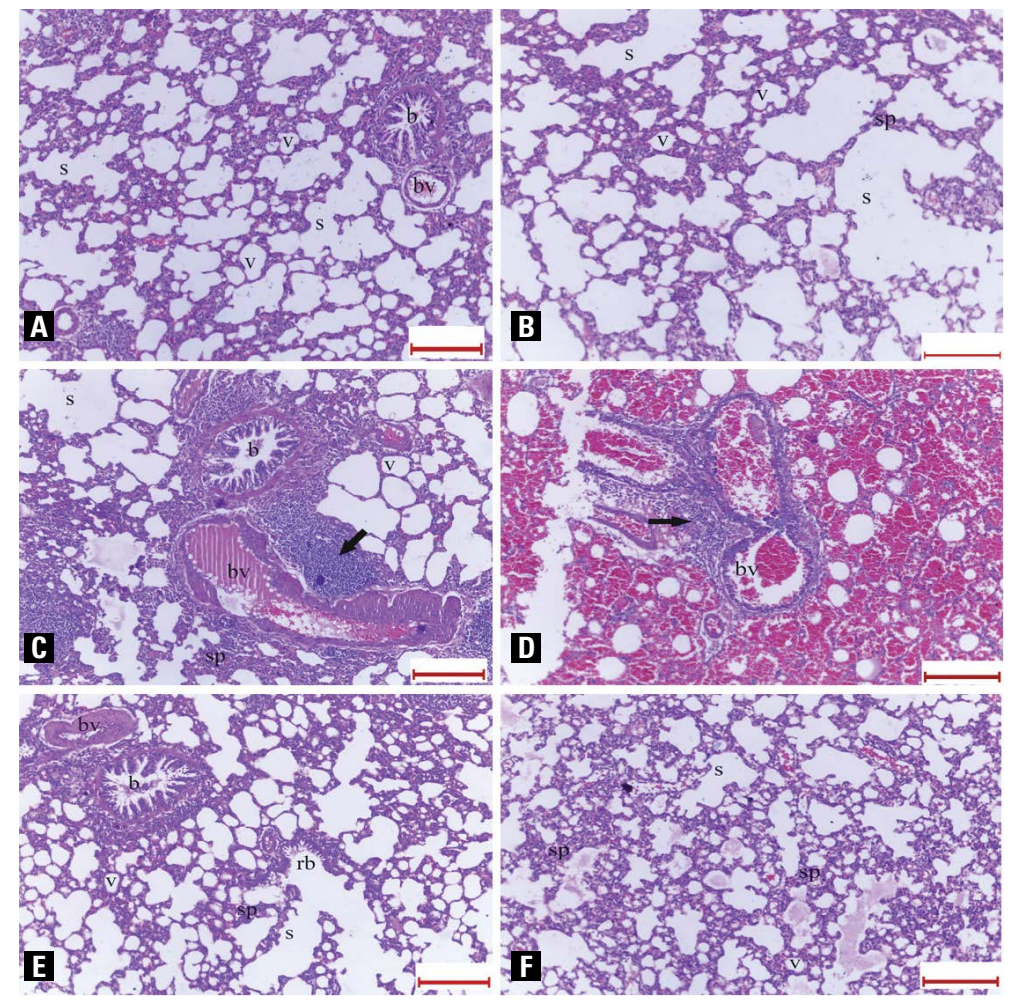

Figure 3. Lung morphology of the different studied groups. Note respiratory bronchiole (rb), terminal bronchiole (b), alveolar sac (s), alveoli (v), interalveolar septa (sp) and blood vessels (bv);

A, B. Normal lung architecture of the control rats; C, D. Inflammatory cellular infiltrations (arrows), thickened interalveolar septa, dilated congested blood vessels and extravasated blood of methotrexate-treated rats; E, F. Normal lung architecture with a pit thickened interalveolar septum and extravasated blood of febuxostat-treated rats. Haematoxylin and eosin, scale bar, $200 \mu \mathrm{m}(\mathrm{A}-\mathrm{F})$. 

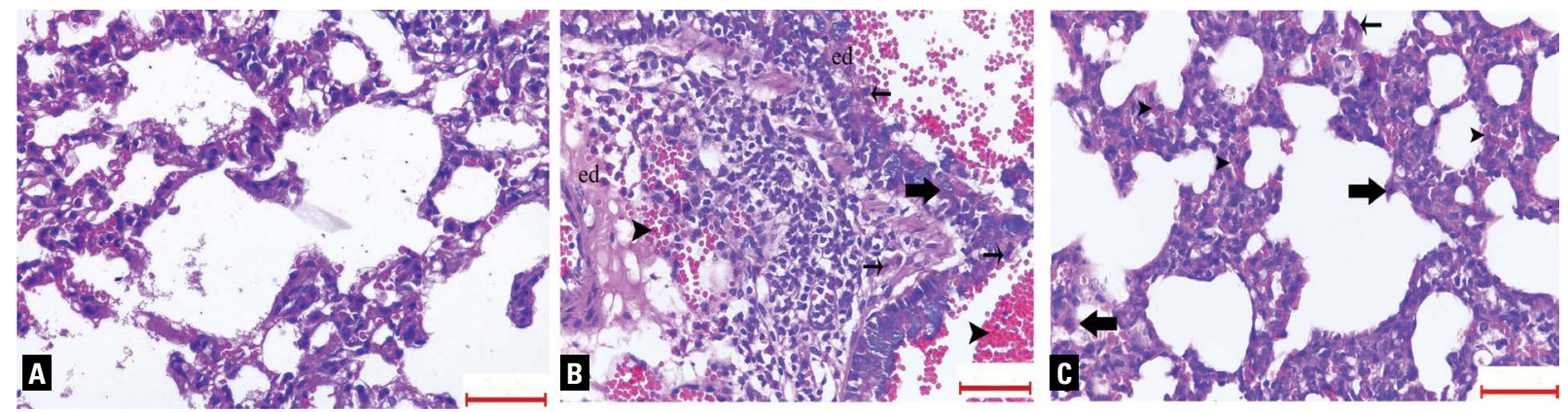

Figure 4. A. Normal lung architecture of the control rats; B. Inflammatory cellular infiltration, extravasated blood (arrowheads), oedema (ed), macrophages (thick arrows) and apoptotic pneumocytes (thin arrows) of methotrexate-treated rats; C. Normal lung architecture with a slight congestion (arrowheads), macrophages (thick arrows) and apoptotic pneumocytes (thin arrows) of febuxostat-treated rats. Haematoxylin and eosin, scale bar, $50 \mu \mathrm{m}(\mathrm{A}-\mathrm{C})$.
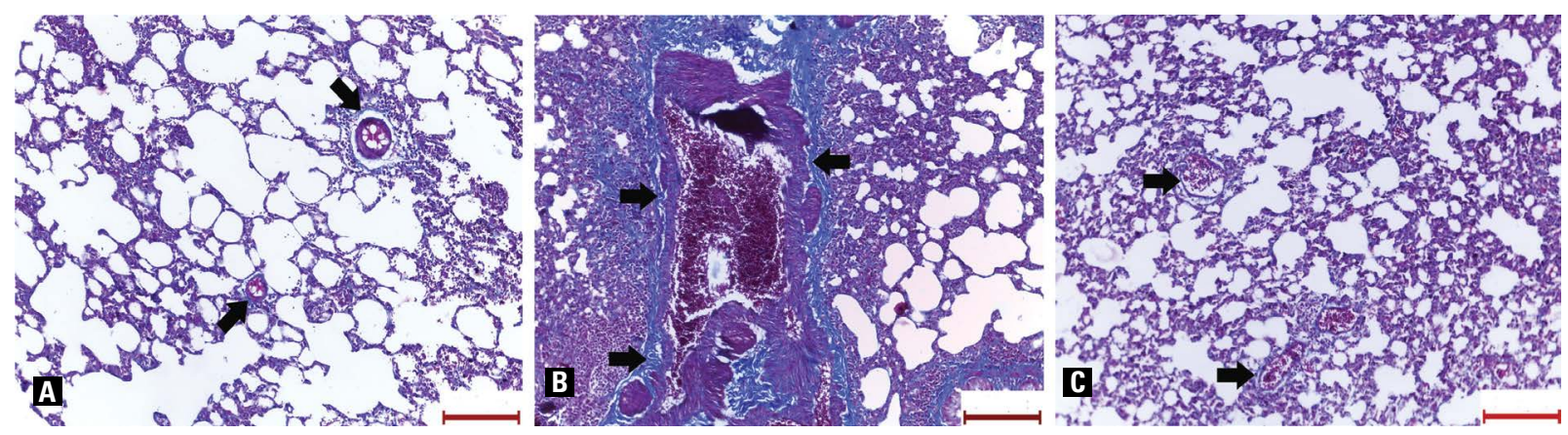

Figure 5. A. The collagen fibres (arrows) in the control rats; B. Increased collagen fibres (arrows) in methotrexate-treated rats; C. Minimal collagen fibres (arrows) in febuxostat-treated rats. Masson's trichrome, scale bar, $200 \mu \mathrm{m}(\mathrm{A}-\mathrm{C})$.

Table 2. Area per cent (\%) of collagen fibres, inducible nitric oxide synthase (iNOS) and cyclooxygenase-2 (COX-2)

\begin{tabular}{llccc}
\hline Group & & Area \% of collagen fibres & COX-2 [cells/mm & iNOS \\
\hline Control & Mean \pm SD & $1.47 \pm 0.54$ & $40 \pm 5$ & $3.1 \pm 0.8$ \\
MTX-treated & Mean \pm SD & $6.0 \pm 0.55$ & $150 \pm 30$ & $18.0 \pm 2.0$ \\
& Versus control & $*$ & $*$ & $*$ \\
& Versus febuxostat-treated & $*$ & $55 \pm 8$ & $5.1 \pm 1.7$ \\
Febuxostat-treated & Mean \pm SD & $2.5 \pm 0.38$ & NS & $*$ \\
& Versus control & NS & $*$ & $*$ \\
\hline
\end{tabular}

*P-value significant. MTX — methotrexate; NS — not significant; SD — standard deviation

ostat-treated group as its fibres contents were comparable to the control group (Fig. 5, Table 2).

\section{Immunohistochemical evaluation}

The COX-2 (cells $/ \mathrm{mm}^{2}$ ) in the control and febuxostat-treated groups were comparable. The immunostained COX-2 cells increased in MTX-treated rats by $>2.5$-fold $(275 \%)$ compared to the control group (Fig. 6, Table 2).
The immunohistochemical reaction of iNOS was poor in both control and febuxostat-treated groups. With use of MXT, the reaction in MXT-treated group became 6-fold higher than in the control group (Fig. 7, Table 2).

\section{Western blot assay}

Nuclear factor kappa B was 2-fold higher than in the control group. With use of febuxostat, NF- $\mathrm{KB}$ be- 


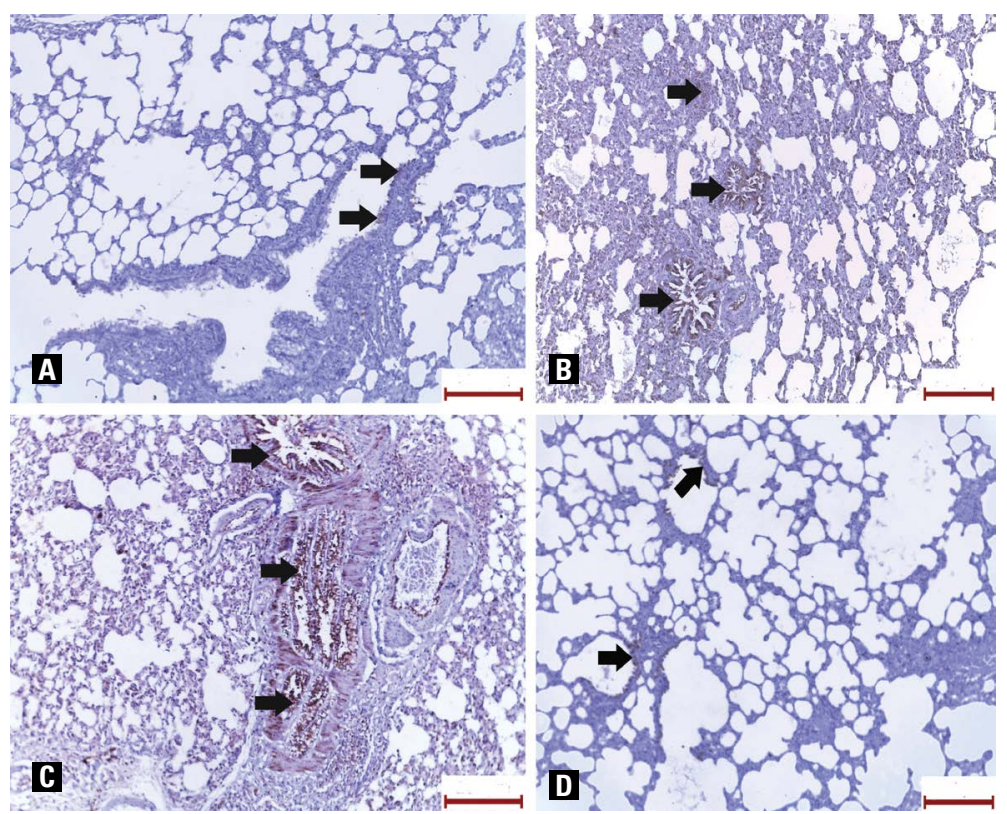

Figure 6. A. Minimal immunostaining of cyclooxygenase-2 (COX-2) (arrows) in the control rats; B, C. Increased staining (arrows) in methotrexate-treated rats; D. Minimal immunostaining of COX-2 (arrows) in febuxostat-treated rats. COX-2 immunostaining, scale bar, $200 \mu \mathrm{m}$ (A-D).

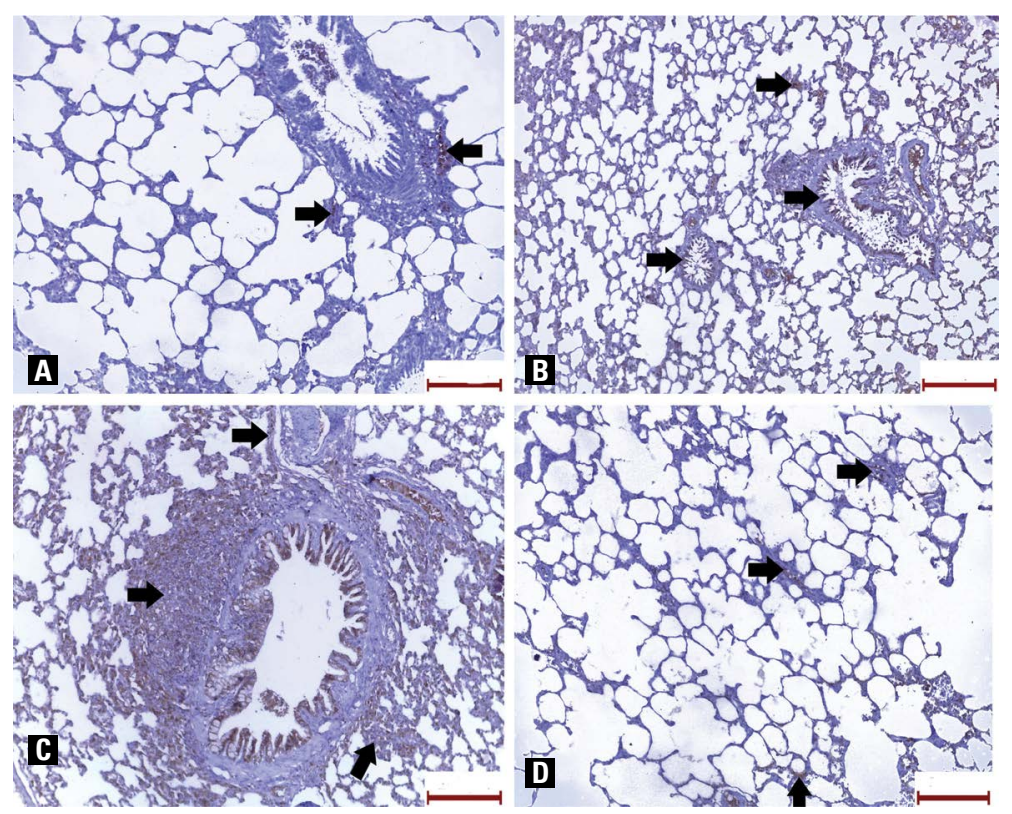

Figure 7. A. Poor immunohistochemically reaction of inducible nitric oxide synthase (iNOS) (arrows) in the control rats; B, C. Increase immunohistochemically reaction of iNOS (arrows) in methotrexate-treated rats; D. Immunohistochemically reaction (arrows) in febuxostat-treated rats. iNOS immunostaining, scale bar, $50 \mu \mathrm{m}$ (A-D).

came 34\% higher than in the control group (Table 3, Fig. 8).

\section{Inflammatory and oxidative/antioxidative markers evaluation (Table 3)}

Interleukin 1 and TNF- $\alpha$ were three-fold higher in MTX-treated group than in the control group.
Improvement was noticed with use of febuxostat as IL-1 and TNF- $\alpha$ were $64 \%$ and $100 \%$ higher than in the control group.

Malondialdehyde was two and half-fold higher than in the control group. With use of febuxostat, MDA became half fold higher than in the control group. 
Table 3. Inflammatory and oxidative/antioxidative markers

\begin{tabular}{|c|c|c|c|c|c|c|c|}
\hline Group & & NF- $\kappa B$ & $\begin{array}{l}\text { IL-1 [pg/mg } \\
\text { protein] }\end{array}$ & $\begin{array}{c}\text { TNF- } \alpha \text { [pg/mg } \\
\text { protein] }\end{array}$ & $\begin{array}{l}\text { MDA [nmoL/mg } \\
\text { protein] }\end{array}$ & $\begin{array}{c}\mathrm{GSH}[\mu \mathrm{g} / \mathrm{mg} \\
\text { protein] }\end{array}$ & $\begin{array}{c}\mathrm{SOD}[\mathrm{nmoL} / \mathrm{mg} \\
\text { protein] }\end{array}$ \\
\hline Control & Mean \pm SD & $1.17 \pm 0.05$ & $149.0 \pm 22.8$ & $123.3 \pm 43.1$ & $1.48 \pm 0.17$ & $1.65 \pm 0.07$ & $8.02 \pm 0.39$ \\
\hline \multirow[t]{3}{*}{ MTX-treated } & Mean \pm SD & $3.80 \pm 0.06$ & $604.0 \pm 17.6$ & $506.6 \pm 28.4$ & $5.22 \pm 0.38$ & $0.66 \pm 0.10$ & $3.07 \pm 0.24$ \\
\hline & Versus control & $*$ & $*$ & $*$ & $*$ & $*$ & $*$ \\
\hline & Versus febuxostat-treated & $*$ & $*$ & $*$ & $*$ & $*$ & $*$ \\
\hline \multirow[t]{3}{*}{ Febuxostat-treated } & Mean \pm SD & $1.57 \pm 0.15$ & $245.0 \pm 21.7$ & $256.6 \pm 19.0$ & $2.22 \pm 0.07$ & $1.69 \pm 0.13$ & $7.10 \pm 0.72$ \\
\hline & Versus control & $*$ & $*$ & $*$ & $*$ & NS & NS \\
\hline & Versus MTX-treated & $*$ & * & * & * & $*$ & $*$ \\
\hline
\end{tabular}

*P-value significant. NF- $\mathrm{KB}$ — nuclear factor kappa B; IL-1 — interleukin 1; TNF- $\alpha$ — tumour necrosis factor alpha; MDA — malondialdehyde; GSH — glutathione; SOD — superoxide dismutase activity; MTX — methotrexate; NS — not significant; SD — standard deviation

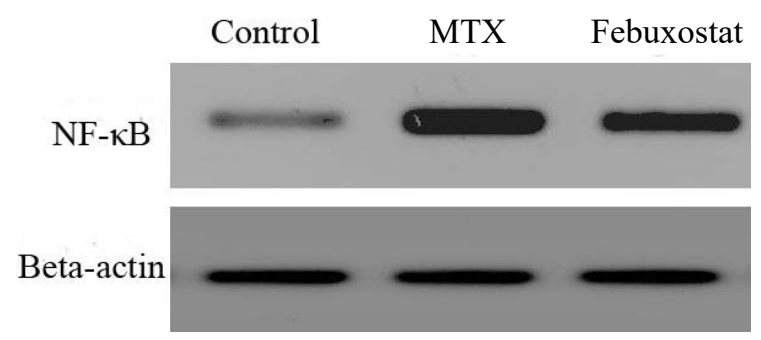

Figure 8. Western blot assay of nuclear factor kappa B (NF- $\mathrm{B})$ in the different groups. NF- $\kappa B$ was higher in methotrexate (MTX)-treated group than the control group. NF- $\kappa B$ was a pit higher in febuxostat-treated rats than the control rats. Beta-actin was used as internal control to measure the relative quantitation of the expression of the target gene.

Superoxide dismutase activity and GSH and decreased by $60 \%$ compared to the control group. Both markers in the control and febuxostat-treated groups were alike.

\section{DISCUSSION}

Body weight of MTX-treated rats decreased by $22 \%$. The loss of weight in rats' model of MTX is due anorexia, cachexia, intestinal mucositis, impairment of absorption and digestive functions, alteration of the gut barrier, and diarrhoea [6, 22].

Methotrexate-treated rats showed a significant increase in the anxiety-like behaviour, expressed by an increase in the number of entries to the dark compartments of the light/dark box, and an increase in the frequency and duration of closed arm entries in the elevated plus maze. The anxiety-like behaviour is explained in mice by the association of MTX with acute brain toxicity and by its ability to induce folate depletion [17]. The anxiety-like behaviour was reported with MTX administration in cancer patients [14]. Much improvement was in febuxostat-treated group which attributed to the antidepressant activity of the febuxostat [20].
Methotrexate-treated rats exhibited an acute inflammatory process (cellular infiltrations, dilated congested blood vessels, thickened interalveolar septa with alveolar oedema). Because of severe congestion, blood extravasation occurred. Adding, apoptotic pneumocytes were detected.

The cause of lung affection in MTX-treated group is multifactorial. The major cause of is the oxidative stress [21]. Oxidative stress is defined as the shift in the balance between oxidants and antioxidants in favour of oxidants [5]. The oxidant marker (MDA) was higher, while the antioxidant markers (SOD and GSH) were lower in MTX-treated rats. It is used for measuring oxidative damage to lipids resulting from free radicals [3]. Analysis of MDA is sufficient to proof and assess the oxidative stress [21]. The elevated MDA is a consequence of the higher level of IL-1 and the toxic dose of MTX [21].

The sterile inflammation in MTX-treated group was caused by an increase of NF- $\kappa B$ (2-fold), IL-1 (3-fold) and TNF- $\alpha$ (3-fold), COX-2 cells (2.5-fold) and iNOS (6-fold). The present neutrophils results in ROS formation and tissue damage through release of chemical mediators [21]. The higher level of IL-1 and higher dose of MTX increase the secretion of proinflammatory cytokines TNF- $\alpha$ [21]. TNF- $\alpha$ is crucially responsible for the pathogenesis of oxidative stress and increases ROS [21]. It regulates growth, proliferation, differentiation, and viability of activated leukocytes [25]. TNF- $\alpha$ also provokes the cellular release of other cytokines, chemokines, or inflammatory mediators [25]. So, excessive TNF- $\alpha$ secretion leads to lung damage by inducing oxidative stress and directly inducing apoptosis.

Fibrosis was observed in MTX-treated group (the collagen fibres content increased 3-fold) as a consequence of oxidative stress and sterile inflammation. 
The third major cause of lung damage in the overdose MTX-treated group is apoptosis. Apoptosis is multifactorial. It is induced by higher level of TNF- $\alpha$ which activates the caspase enzyme system [21]. Apoptosis is also induced by lipid peroxidation (higher MDA) as MDA is the end product of the lipid peroxidation process [5]. Lipid peroxidation results in organ and cell damage [21]. The increased observed apoptosis results in excessive release of cytokines and enhanced ROS which finally damage the lung tissue [21].

Another possible cause of lung injury in MXT-treated group is the elevation of iNOS. The nitric oxide-derived from iNOS appears to induce inflammatory infiltration and oxidative stress. These events create a deleterious environment that ultimately leads to cellular damages. It seems that disruption of COX-2 activity aggravates the resultant lung damage [18].

With the use of febuxostat, the normal lung architecture was observed with a bit thickened interalveolar septum and extravasated blood. The collagen fibres content was minimal. Decrement of oxidative stress and sterile inflammation (COX-2 cells and iNOS were comparable to the control group. NF-kB, IL-1 and TNF- $\alpha$ became higher by $34 \%, 64 \%$ and $100 \%$ ). The protective role of febuxostat is due to its anti-inflammatory and antioxidant features [27]. Febuxostat showed anti-inflammatory effects in different experimental models [15]. Its antioxidative stress effect was proved in in the rat model of renal ischaemia-reperfusion injury [36].

\section{CONCLUSIONS}

In conclusion, the overdose of MTX displays inflammatory lung affection with residual fibrosis. It induces lung affection through oxidative stress, apoptosis and sterile inflammation. With the use of febuxostat, the normal lung architecture was preserved with a little structural affection or fibrotic residue. Febuxostat exerts its lung protection through its anti-inflammatory and antioxidant features.

\section{Acknowledgements}

Acknowledgements to all my colleagues who support me during this work.

\section{REFERENCES}

1. Alamir I, Boukhettala N, Aziz M, et al. Beneficial effects of cathepsin inhibition to prevent chemotherapy-induced intestinal mucositis. Clin Exp Immunol. 2010; 162(2): 298-305, doi: 10.1111/j.1365-2249.2010.04220.x, indexed in Pubmed: 20731673.
2. Arpag H, Gül M, Aydemir $Y$, et al. Protective effects of alpha-lipoic acid on methotrexate-induced oxidative lung injury in rats. J Invest Surg. 2018; 31(2): 107-113, doi: 10.1080/08941939.2017.1296513, indexed in Pubmed: 28340320.

3. Atuğ Özcan SS, Ceylan I, Ozcan E, et al. Evaluation of oxidative stress biomarkers in patients with fixed orthodontic appliances. Dis Markers. 2014; 2014: 597892, doi: 10.1155/2014/597892, indexed in Pubmed: 24864131.

4. Barrera P, Laan RF, van Riel PL, et al. Methotrexate-related pulmonary complications in rheumatoid arthritis. Ann Rheum Dis. 1994; 53(7): 434-439, doi: 10.1136/ ard.53.7.434, indexed in Pubmed: 7944614.

5. Birben E, Sahiner UM, Sackesen C, et al. Oxidative stress and antioxidant defense. World Allergy Organ J. 2012; 5(1): 9-19, doi: 10.1097/WOX.0b013e3182439613, indexed in Pubmed: 23268465.

6. Boukhettala N, Leblond J, Claeyssens S, et al. Methotrexate induces intestinal mucositis and alters gut protein metabolism independently of reduced food intake. Am J Physiol Endocrinol Metab. 2009; 296(1): E182-E190, doi: 10.1152/ajpendo.90459.2008, indexed in Pubmed: 18984853.

7. Bourin $M$, Hascoët $M$. The mouse light/dark box test. Eur J Pharmacol. 2003; 463(1-3): 55-65, doi: 10.1016/s00142999(03)01274-3.

8. Bradford M. A rapid and sensitive method for the quantitation of microgram quantities of protein utilizing the principle of protein-dye binding. Anal Biochem. 1976; 72(1-2): 248-254, doi: 10.1016/0003-2697(76)90527-3.

9. Carson C, Cannon G, Egger M, et al. Pulmonary disease during the treatment of rheumatoid arthritis with low dose pulse methotrexate. Semin Arthritis Rheum. 1987; 16(3): 186-195, doi: 10.1016/0049-0172(87)90021-7.

10. Charan J, Biswas T. How to calculate sample size for different study designs in medical research? Indian J Psychol Med. 2013; 35(2): 121-126, doi: 10.4103/02537176.116232, indexed in Pubmed: 24049221.

11. Conway R, Low C, Coughlan RJ, et al. Methotrexate use and risk of lung disease in psoriasis, psoriatic arthritis, and inflammatory bowel disease: systematic literature review and meta-analysis of randomised controlled trials. BMJ. 2015; 350: h1269, doi: 10.1136/bmj.h1269, indexed in Pubmed: 25770113.

12. Dhouib $H$, Jallouli $M$, Draief $M$, et al. Oxidative damage and histopathological changes in lung of rat chronically exposed to nicotine alone or associated to ethanol. Pathol Biol (Paris). 2015; 63(6): 258-267, doi: 10.1016/j.patbio.2015.10.001, indexed in Pubmed: 26586280.

13. Dignass A, Van Assche G, Lindsay JO, et al. The second European evidence-based Consensus on the diagnosis and management of Crohn's disease: Current management. J Crohns Colitis. 2010; 4(1): 28-62, doi: 10.1016/j. crohns.2009.12.002, indexed in Pubmed: 21122489.

14. Elens I, Dekeyster E, Moons L. Methotrexate affects cerebrospinal fluid folate and tau levels and induces late cognitive deficits in mice. Neuroscience. 2019; 404: 62-70, doi: 10.1016/j.neuroscience.2019.01.024, indexed in Pubmed: 30703509.

15. Fahmi AN, Shehatou GS, Shebl AM. Febuxostat protects rats against lipopolysaccharide-induced lung inflammation 
in a dose-dependent manner, Naunyn Schmiedebergs. Arch Pharmacol, 389(3), pp. 2016; 389(3): 269-278, doi: 10.1007/s00210-015-1202-6, indexed in Pubmed: 26713331.

16. Festing MF, Altman DG. Guidelines for the design and statistical analysis of experiments using laboratory animals. ILAR J, 43(4), pp. 2002; 43(4): 244-258, doi: 10.1093/ ilar.43.4.244, indexed in Pubmed: 12391400.

17. François $M$, Takagi $K$, Legrand $R$, et al. Increased ghrelin but low ghrelin-reactive immunoglobulins in a rat model of methotrexate chemotherapy-induced anorexia. Front Nutr. 2016; 3: 23, doi: 10.3389/fnut.2016.00023, indexed in Pubmed: 27508207.

18. Fukunaga K, Kohli P, Bonnans C, et al. Cyclooxygenase 2 plays a pivotal role in the resolution of acute lung injury. J Immunol. 2005; 174(8): 5033-5039, doi: 10.4049/ jimmunol.174.8.5033, indexed in Pubmed: 15814734.

19. Huang $C$, Hsu $P$, Hung $Y$, et al. Ornithine decarboxylase prevents methotrexate-induced apoptosis by reducing intracellular reactive oxygen species production. Apoptosis, 2005; 10(4): 895-907, doi: 10.1007/s10495-005-2947-z, indexed in Pubmed: 16133879.

20. Karve AV, Jagtiani S, Chitnis KSA. Evaluation of effect of allopurinol and febuxostat in behavioral model of depression in mice. Indian J Pharmacol. 2013; 45(3): 244-247, doi: 10.4103/0253-7613.111922, indexed in Pubmed: 23833366.

21. Kurt A, Tumkaya L, Turut $H$, et al. Protective effects of infliximab on lung injury induced by methotrexate. Arch Bronconeumol. 2015; 51(11): 551-557, doi: 10.1016/j. arbr.2015.05.012.

22. Leblond J, Le Pessot F, Hubert-Buron A, et al. Chemotherapy-induced mucositis is associated with changes in proteolytic pathways. Exp Biol Med (Maywood). 2008; 233(2): 219-228, doi: 10.3181/0702-RM-49, indexed in Pubmed: 18222977.

23. Misra $H$, Fridovich I. The role of superoxide anion in the autoxidation of epinephrine and a simple assay for superoxide dismutase. J Biol Chem. 1972; 247(10): 3170-3175, doi: 10.1016/s0021-9258(19)45228-9.

24. Mohamed DI, Khairy E, Tawfek SS, et al. Coenzyme Q10 attenuates lung and liver fibrosis via modulation of autophagy in methotrexate treated rat. Biomed Pharmacother. 2019; 109: 892-901, doi: 10.1016/j.biopha.2018.10.133, indexed in Pubmed: 30551543.

25. Nair MP, Mahajan S, Reynolds JL, et al. The flavonoid quercetin inhibits proinflammatory cytokine (tumor necrosis factor alpha) gene expression in normal peripheral blood mononuclear cells via modulation of the NF-kappa beta system. Clin Vaccine Immunol. 2006; 13(3): 319-328, doi: 10.1128/CVI.13.3.319-328.2006, indexed in Pubmed: 16522772.

26. Ohbayashi M, Kubota S, Kawase A, et al. Involvement of epithelial-mesenchymal transition in methotrexate-induced pulmonary fibrosis. J Toxicol Sci. 2014; 39(2):
319-330, doi: 10.2131/jts.39.319, indexed in Pubmed: 24646714

27. Pacher P, Nivorozhkin A, Szabó C. Therapeutic effects of xanthine oxidase inhibitors: renaissance half a century after the discovery of allopurinol. Pharmacol Rev. 2006; 58(1): 87-114, doi: 10.1124/pr.58.1.6, indexed in Pubmed: 16507884

28. Pui CH, Pei D, Pappo AS, et al. Treatment outcomes in black and white children with cancer: results from the SEER database and St Jude Children's Research Hospital, 1992 through 2007. J Clin Oncol. 2012; 30(16): 2005-2012, doi: 10.1200/JCO.2011.40.8617, indexed in Pubmed: 22547602.

29. Rahman I, Kode A, Biswas SK. Assay for quantitative determination of glutathione and glutathione disulfide levels using enzymatic recycling method. Nat Protoc. 2006; 1(6): 3159-3165, doi: 10.1038/nprot.2006.378, indexed in Pubmed: 17406579.

30. Ramos-Vara JA, Kiupel M, Baszler T. Suggested guidelines for immunohistochemical techniques in veterinary diagnostic laboratories. J Vet Diagn Invest. 2008; 20(4): 393-413, doi: 10.1177/104063870802000401, indexed in Pubmed: 18599844.

31. Robinson PC, Dalbeth N. Febuxostat for the treatment of hyperuricaemia in gout. Expert Opin Pharmacother. 2018; 19(11): 1289-1299.

32. Sakthiswary R, Suresh E. Methotrexate in systemic lupus erythematosus: a systematic review of its efficacy. Lupus. 2014; 23(3): 225-235, doi: 10.1177/0961203313519159, indexed in Pubmed: 24399812.

33. Sathi N, Chikura B, Kaushik VV, et al. How common is methotrexate pneumonitis? A large prospective study investigates. Clin Rheumatol. 2012; 31(1): 79-83, doi: 10.1007/s10067-011-1758-6, indexed in Pubmed: 21638023

34. Sivalingam VN, Duncan WC, Kirk E, et al. Diagnosis and management of ectopic pregnancy. J Fam Plann Reprod Health Care. 2011; 37(4): 231-240, doi: 10.1136/jfprhc-2011-0073, indexed in Pubmed: 21727242.

35. Suvarna SK, Layton C, Bancroft JD. Bancroft's theory and practice of histological techniques. Eighth edition. Oxford, Elsevier 2019.

36. Tsuda H, Kawada N, Kaimori Jy, et al. Febuxostat suppressed renal ischemia-reperfusion injury via reduced oxidative stress. Biochem Biophys Res Commun. 2012; 427(2): 266-272, doi: 10.1016/j.bbrc.2012.09.032, indexed in Pubmed: 22995295.

37. Walf AA, Frye CA. The use of the elevated plus maze as an assay of anxiety-related behavior in rodents. Nat Protoc. 2007; 2(2): 322-328, doi: 10.1038/nprot.2007.44, indexed in Pubmed: 17406592.

38. Zhu H, Deng FY, Mo XB, et al. Pharmacogenetics and pharmacogenomics for rheumatoid arthritis responsiveness to methotrexate treatment: the 2013 update. Pharmacogenomics. 2014; 15(4): 551-566, doi: 10.2217/pgs.14.25, indexed in Pubmed: 24624921. 